\title{
How Albanian Private Universities can use Game Theory for Optimization of Scholarship Offers.
}

\author{
Dr. Llambrini Sota ${ }^{1}$, Prof. Dr. Fejzi Kolaneci ${ }^{2}$
}

\begin{abstract}
:
There are 46 private universities in Albania. We believe that the tuition fee and scholarship for high GPA students are two important components of the competition between private universities. This study is a first attempt in applying Game Theory for optimization of scholarship offers by Albanian private universities during academic years. There is a conflict between the utility functions of shareholders to maximize their profits and the utility functions of the students enrolled in private universities to minimize their expenses. The best solution of the conflict is a Nash equilibrium or Bayes-Nash-Harsanyi equilibrium, respectively. We prove the existence of these equilibria. However, there exist many equilibria, depending of private information. We interpret the Boyes-Nash-Harsanyi equilibrium solution as the set of possible rational concessions made by all players.
\end{abstract}

Keywords: private universities, scholarship, optimization, game theory, Nash equilibrium.

\section{Introduction}

Actually, there are 46 private universities in Albania. Strengthening the position of private universities in the Republic of Albania in the actual conditions is a complicated problem involving several variables: profit, risk, individual goals and preferences, teaching and scientific levels, financial support, competitors, social and legal rules, corruption, etc. In this study, using Game Theory, Probability and Mathematical Statistics, we will try to find an optimal scholarship strategy in the private universities of Albania during the academic years. We apply Nash equilibrium, Bayes-Nash-Harsanyi equilibrium, and subjective equilibrium.

\section{Main components of competition between private universities are:}

1. Tuition fee

2. Scholarship for high GPA students, financial support for special cases

3. Degree of Bachelor of Science, Master Degrees, PhD. Degrees that are offered

${ }^{1}$ University Pavarësia, Vlora, Albania.

${ }^{2}$ University of New York Tirana, Albania. 
4. Quality of teaching

5. Employment percentage of graduates

6. Quality of the library, availability of electronic data, computerization

7. Campuses and buildings

8. Research, international or natural scientific conferences, and publications

9. Honorary doctors and famous alumni

10. International cooperation.

The number of new students enrolled in a private university during different academic years is a random variable, depending on a plethora of economic, political, social, competitive, psychological and personal factors. In the following, we investigate the strategic game as a model of new students enrolled at Albanian private universities.

A game in strategic (or normal) form has three components: the set of all players which we assume to be finite $\{1,2, \ldots, m\}$, the pure strategy space $S_{k}$ for each player $\mathrm{K}$, and the utility functions $\mathrm{U}_{\mathrm{k}}=\mathrm{U}_{\mathrm{k}}(\mathrm{s})$ for each profile of strategies (situation) $\mathrm{s}=\left(\mathrm{s}_{1}, \mathrm{~s}_{2}, \ldots \mathrm{s}_{\mathrm{m}}\right)$. Each player's objective is to maximize his own utility function, and this may involve "helping" or "hurting" the other players. For economists, the most familiar interpretation of strategies may be as choices of prices or output levels.

A mixed strategy is a probability distribution over pure strategies. We assume that each player's randomization over the space of pure strategies is independent of those of his (her) opponents. The utility functions to a profile of mixed strategies are the expectations (mathematical expectations) of the corresponding pure-strategy utility functions.

According to A. N. Kolmogorov, bargaining is a random process to settle disputes and reach mutually beneficial agreements. Typical situations of bargaining are characterized by two or more participants (agents) who have common interest in cooperating, but conflicting interests in the way of doing so. The outcomes of bargaining depend on agents' attitudes towards their bargaining items and their mathematical expectations from the realized bargaining. The representation of a of a bargainer's attitudes in Game Theory is implicit via utility functions.

The Nash bargaining game is a game in strategic form used to model bargaining interactions between the players. This game was first suggested by John Forbes Nash Jr. in his 1950 paper "The bargaining Problem". Nash idealizes the bargaining process by assuming that players are rational.

The rational behavior of each player is individual behavior that satisfies the following axioms: 
1. A player uses his (her) strategy on the basis of information concerning the strategy sets and the utility functions of all players.

This axiom means that players don't use irrelevant information for his (her) strategy.

2. In choosing his (her) strategy, each player assumes that the other players are rational in the same way as he (she) him(her)self is rational.

This axiom implies a sort of symmetry. All players are rational in the same way. It is part of the rational behavior of the individual players to recognize and take into account the rationality of other players.

3. If some strategy is the rational strategy for an individual player, then this strategy can be correctly predicted by other players.

This axiom assumes that all players have the same and full information about the situation, i.e. each player knows the strategy sets and utility functions of other players as well as his (her) own. Each player can predict the possible strategies that will be taken by other players, but he (she) also knows, in conformity with axiom 2, that the other players can predict his (her) own strategy as well.

4. Being able to predict the strategies to be taken by other players, a player's own strategy maximizes his (her) utility function corresponding to the predicted strategies of other players.

5. A strategy is rational if the player, after having observed the strategies taken by other players and the outcome of the game, does not regret the strategy he (she) made.

We clarify that the expression "the player does not regret" means that if the player was again put in the same situation, he (she) would make the same strategy.

We assume the game to be non cooperative. This means that each player makes his (her) own strategy without communicating with others. In other words, when player $\mathrm{K}$ is to make his (her) strategy $\mathrm{s}_{\mathrm{k}}$, he (she)has not received any information from the other players about their strategies. On the other hand, we assume that each player is fully informed about the strategy sets and utility functions of all players.

The non cooperative game concept was used and investigated by Nash (1951). It is described by him as a concept relevant to the Game Theory "based on the absence of coalitions in that it is assumed that each participant acts independently, without collaboration or communication with any of the others".

Thus, each player attempts to maximize a utility function $\mathrm{u}_{\mathrm{k}}=\mathrm{u}_{\mathrm{k}}\left(\mathrm{s}_{1}, \mathrm{~s}_{2}\right.$, $\ldots \mathrm{s}_{\mathrm{m}}$ ) of which he (she) does not control all variables. This is not a maximum problem of Calculus, but a peculiar and disconcerting mixture of several 
conflicting maximum problems. This kind of problem is nowhere dealt within Calculus.

\section{The Nash Equilibrium}

According to Nash, the utility functions of each player (of the bargaining game) satisfy the following axioms:

1. Effectiveness: The bargaining is applicable in practice if none of the players lose from it.

2. Symmetry: If the players in conflict have symmetric positions in the bargaining scheme, then they must gain equal utility (profit).

3. Pareto's Optimality: In the bargaining game, players cannot simultaneously and unlimitedly increase their profits. This axiom expresses the logic of the bargaining: in a reasonable bargaining it is impossible for all the players to increase their profits unlimitedly.

4. Monotony: The players in the recent bargaining must respect all previous bargaining, that are broader (more general) than the recent one.

5. Invariance: of the utility function with respect to affine transformation of the coordinative system.

6. Independence: of utility function from irrelevant alternatives.

\section{A Nash equilibrium of a bargaining game is a profile of strategies (or situation)}

$\mathrm{S}=\left(\mathrm{s}_{1}, \mathrm{~s}_{2}, \ldots, \mathrm{s}_{\mathrm{k}}, \ldots \mathrm{s}_{\mathrm{m}}\right)$

such that each player's strategy $s_{k}$ is an optimal response to the other $(\mathrm{m}-1)$ players' strategies. It would survive an announcement test: if all $\mathrm{m}$ players announced their strategies simultaneously, nobody would want to reconsider. The Nash equilibrium is a self-enforcing agreement. That is, an explicit or implicit agreement that, once reached by $\mathrm{m}$ players, does not need any external means of enforcement, because it is in the self interest of each player to follow the agreement if the others do. Nash equilibria are consistent predictions of how the bargaining game will be played, in the sense that if all players predict that a particular Nash equilibrium will occur, then no player has an incentive to play differently. theorem:

Using Kakutani's fixed point theorem, Nash (1951) proved the following

Every finite strategic form bargaining game has a mixed-strategy Nash equilibrium 
Nash idealized the bargaining problem by assuming that all players are rational, that each player can accurately compare his (her) desires for various things, that players are approximately equal in bargaining skills, and that each of them has full knowledge of the preferences of the others.

The fundamental principle in modern Game Theory is that any theory or social plan that predicts or prescribes behavior of all players in a bargaining game must designate a Nash equilibrium, if this theory or social plan is to be understood correctly by all the players and is not to impute irrational behavior to any player at any point in space-time. Rational-choice analysis (or the assumption of perfect rational players) is certainly imperfect as a description of real human behavior.

In 1994 the Nobel Prize in Economic Sciences was awarded to John F. Nash Jr., John C. Harsanyi, and Reinhard Selten. A detailed biography of Nash has been written by Silvia Nasar (1998). Nash's theory of non-cooperative games was a major turning point in the history of economic thought.

Today there is an important and general problem of non-complete information in economics, social sciences, etc. A serious difficulty with the existence and numerical approximation of Nash equilibrium is the assumption that "the beginning of bargaining game must be a point in time when all players have the same information". This restriction can be awkward for modeling situations where players have long-standing differences in information. Harsanyi (1967-1968) showed how to avoid this difficulty by constructing Bayesian game models of incomplete information. The informational differences in players are not simply ad-hoc phenomena, but can be explained by differences in players' experience. According to A. N. Kolmogorov, players know something privately, which has a major impact on his preferences. Harsanyi (1973) proved the existence of randomized Bayes-Nash-Harsanyi equilibria which depends critically by something that the players know privately.

Nash's non-cooperative game theory, Nash equilibria, and Bayes-NashHarsanyi equilibria are abstract mathematical approaches for economic, social or political analysis. They are not economical or political analysis themselves.

Selten was cited for his work in equilibrium refinements, which takes the point of view that the requirements of the Nash equilibrium are necessary conditions, but are not sufficient conditions for advice to perfectly rational players in the bargaining game. Selten $(1965,1975)$ showed that for many bargaining games there exist too many Nash equilibria.

Harsanyi (1973) showed that any randomized Bayes-Nash-Harsanyi equilibrium of a strategic-form game could be interpreted as a pure (nonrandomized) equilibrium of a very similar Bayesian game, in which each player has some independent private information that affects the player's preferences. 
Today, the theory of non-cooperative games that Nash, Harsanyi, and Selten created can help us to better understand the problems of conflict and cooperation in virtually any economic, social or political institution. We accept and use non-cooperative game theory as a core analytical methodology alongside price theory. We consider the non-cooperative game related to the scolarships offered by a private university's shareholders and Academic Council for High School Students with GPA 8-9 or 9-10.For example, if we continue with data from the same university we discussed before (UNYT), we would say that the players are the President of UNYT, The Vice President, the Academic Council of this university, and the families of all High School graduates in Albania, Kosovo, etc, with GPA 8-9 or 9-10. The players have common interest and desire for collaboration (high quality of teaching-education-research programs offered by the university's academic staff, very good books, the degree of Bachelor of Science delivered by the university, etc). But there is also a conflict between the players: the preference of shareholders is to maximize their profits, but the families of the enrolled students prefer to minimize their expenses.

Following Kolmogorov, we interpret the solution of non-cooperative bargaining game (in the sense of Nash-Bayes-Harsanyi) as the set of concessions made by all players. The best (optimal) solution is Nash equilibrium or BayesNash-Harsanyi equilibrium, respectively.

We apply the definition of subjective probability (according to Mizes, Hinchin and Kolmogorov). This definition requires that all shareholders, members of Academic Council of the university, the Academic coordinator, the Finance Office and Marketing Office, evaluate independently of each-other the best (optimal) strategy related to fellowship offered by the university for high GPA High School graduates. These data will be mathematically analyzed by a statistics expert, in order to define the optimal strategy (in the sense of Nash equilibrium).

The Marketing office should conduct inquiries with students to be graduated from the private and public High Schools of Albania. Our intention is to learn their reaction towards the proposals of the private university for discounts of tuition fees.

\section{Bayesian game and Bayes-Nash-Harsanyi equilibrium.}

In game theory, a Bayesian game is one in which information about the other players (i.e., strategies or utility functions) is incomplete. Such game is called Bayesian because of the probabilistic analysis (Bayes approach) inherent in the game. Players have initial beliefs about the "type" of each player. A belief is a probability distribution over the possible types for an arbitrary player. Players can update their beliefs according to the Bayes Rule as play takes place in the 
game, i.e. the belief a player holds about another player's type might change on the basis of the actions they have played. The lack of information held by players and mathematical modeling of beliefs mean that Beyesian games are also used to analyze imperfect information interactions. Following A. N. Kolmogorov and J. C. Harsanyi , a Bayesian game can be modeled by introducing Nature as a player in the game. Nature assigns a random variable to each player could take values of type for each player and associating probability density functions within those types. A "player type" is his/her private information, which is relevant to the player's decision making. This private information can include, in addition to the player's utility functions, his/her beliefs about other players' utility functions about what other players believe his/her beliefs are, and so on. In many applications, the player's types are identified with their utility functions. Harsanyi assumed that the players' types $\theta_{1}, \theta_{2}, \ldots, \theta_{1} \ldots, \theta_{n}$, are drawn by some objective probability distribution $\mathrm{p}\left(\theta_{1}, \theta_{2}, \ldots, \theta_{\mathrm{i}}, \ldots \theta_{\mathrm{n}}\right)$. We assume that the marginal probabilities $\mathrm{p}_{\mathrm{i}}\left(\theta_{\mathrm{i}}\right)$ are strictly positive for all $\mathrm{i}=1,2, \ldots, \mathrm{n}$. Each player's choice of individual strategy depends on his/her type. Kolmogorov-Harsanyi's approach to modeling a Bayesian game in such a way allows games of incomplete information to become games of imperfect information! In a Bayesian game, the incompleteness of information means that at least one player is unsure of the type and utility function of another player (where a belief is a probability distribution over the possible types for an arbitrary player) and can update their beliefs using Bayes Rule as play takes place in the game. Therefore, the belief a player holds about another player's type might change on the basis of strategies they have played.

In a Bayesian game, it is necessary to specify the strategy spaces, type spaces, utility functions and beliefs for every player. To complete the description of a Bayesian game, we must specify:

- $\quad$ A set of players $1,2, \ldots, 1, \ldots n$;

- A pure strategy space $S_{i}=\left\{S_{i}\right\}$ for each player $i\left(S_{i}\right.$ represents the choices of actions for player i);

- $\quad$ A mixed strategy space $\Sigma_{\mathrm{i}}=\left\{\delta_{\mathrm{i}}\right\}$ for each player i;

- A set $\Omega=\{\omega\}$ of the states of the Nature;

- A utility function $\mathrm{u}_{\mathrm{i}}=\mathrm{u}_{\mathrm{i}}\left(\mathrm{s}_{1}, \mathrm{~s}_{2}, \ldots, \mathrm{s}_{\mathrm{n}}, \theta_{1}, \theta_{2}, \ldots, \theta_{\mathrm{m}}, \omega\right)$ for each player $\mathrm{i}$, where $\theta_{1}$ denotes the type of player $i$.

The strategyspaces $\mathrm{S}_{\mathrm{i}}, \Sigma_{\mathrm{i}}$, utility functions $\mathrm{u}_{\mathrm{i}}$, possible types $\theta_{1}, \theta_{2}, \ldots, \theta_{\mathrm{i}} \ldots, \theta_{\mathrm{n}}$, and prior probability distributions $\mathrm{p}\left(\theta_{1}, \theta_{2}, \ldots, \theta_{\mathrm{i}}, \ldots \theta_{\mathrm{n}}\right)$ are "common knowledge" of the Bayesian game. Any initial private information that a player i may have is included in the description of his/her type. The strategy spaces are abstract mathematical objects. The utility functions $\mathrm{u}_{1}, \mathrm{u}_{2}, \ldots \mathrm{u}_{\mathrm{n}}$ of the Bayesian players can be interpreted (in many cases) as mathematical expectations over moves by 
Nature (that is, as the expectation random variables). These expectations are not known by any player, when the players pick their pure or mixed strategies. Let $\theta_{i}$ denote the mixed or pure strategy player $i$ chooses when its type is $\theta_{i}$. If player iknows the strategies $\delta_{j}\left(\theta_{j}\right), 1 \leq j \leq n, j \neq i$ of the other players, then player $\mathrm{i}$ could use his/her beliefsp $\left(\theta_{1}, \ldots \theta_{1-1}, \theta_{i+1}, \ldots \theta_{\mathrm{n}} \theta_{\mathrm{i}}\right)$ to compute the expected utility to each choice and thus to find (calculate) his/her optimal response $\delta_{i}\left(\theta_{i}\right)$.

Many games of interest in economics, political sciences, natural sciences, technical sciences and technologies have incomplete information (are Bayesian).

We play a Bayesian game with enrolled students in private universities. Harsanyi's Bayesian equilibrium (or Bayes-Nash-Harsanyi equilibrium) is precisely the Nash equilibrium of the incomplete information (or Bayesian) game.

\section{Definition (Harsanyi, 1973)}

A Bayesian-Nash-Harsanyi equilibrium is defined as a strategy profile and beliefs specified for each player about types of the other players that maximizes the mathematical expectation of utility function for each player, given their beliefs about the other players' types and given the pure (or mixed) strategies played by the other players. Kolmogorov and Harsanyi assume that the players' types are statistically independent, the players have the same prior beliefs about the probability distribution of nature's moves, and the nature's moves represent random events.

The existence of a Bayes-Nash-Harsanyi equilibrium is an immediate consequence of the Bayes Principle, Nash Existence Theorem and Fort Fixed Point Theorem.

Harsanyi (1973) has shown that mixed-strategy Nash equilibria of complete information games can usually be interpreted as the limit when $\varepsilon \rightarrow 0^{+}$of $\varepsilon-$ pure strategy equilibria of slightly perturbed games of incomplete (or imperfect) information:

The probability distribution over strategies induced by the pure-strategy equilibria of the perturbed Bayesian game converges to the probability distribution of the mixed-strategy equilibrium of the unperturbed complete information game.

Our view is that, games of complete information are an idealization, as players typically have at least a slight amount of incomplete information about the other player's' utility functions or objectives. One important consequence of this view, as Harsanyi's argument proves, is that the distribution between pure and mixed strategies may be artificial.

John C. Harsanyi and R. B. Myerson showed that the presence of incomplete information would tend to introduce inefficiencies in the bargaining game. For 
example, a student may have an incentive to reject a price offered by a private university that is below his/her valuation for that institution, in hope of obtaining a better price offered by another private university. If the Bayes-NashHarsanyi equilibrium of a bargaining game is inefficient, then the evaluation of experts (scientists) can influence the efficiency of the outcome. It was realized (by D. Fudenberg and J. Tirole 1983, R. Carmona 1984...) that the private information of players has an important impact in the existence of the BayesNash-Harsanyi equilibria. The idea of using learning-type process to explain Bayes-Nash-Harsanyi equilibrium in strategic games goes back to Nash and Vorobiev. We make the hypothesis of learning history: Players must have enough experience to learn how their opponents play and the outcomes must be convergent. Moreover, we assume that there is a large sample of players ( $\mathrm{n}>40)$ who are randomly selected. Therefore, we can apply in our study Kolmogorov's Central Limit Theorem. Recently, some well-known economists like E. Maskin, R. J. Aumann, H. Lucas Jr., et have argued that the evolution can be taken as a metaphor for learning process in Bayesian games.

\section{Subjective game and subjective equilibrium}

The concept of Nash equilibrium (1950) and its extensions to BayesNash-Harsanyi equilibrium (1967) and to correlated equilibrium by Aumann (1974) have become the main tools for modeling and analyzing strategic interactions under uncertainty. These elegant concepts and corresponding theories give researchers the ability to make predictions in uncertain environments. In applying these concepts and theories, researchers make the following assumptions about the players in a game.

1. A Complete Mathematical Model. Each player has complete information about the utility functions and strategies of his/her opponents. That is, all players possess objective knowledge of the game.

2. Correct Common Prior Probability Distributions. When such complete information is missing, the players assign correct prior probability distributions to all unknown parameters (random variables) of the mathematical model.

3. A Closed Mathematical Model. Each player assumes that his/her opponents model the game exactly and correctly as he/she does and, moreover, they too assign the same correct prior probability distributions to all unknown parameters of the mathematical model.

Such knowledge is often not available. In many applications the above assumptions are unrealistic and, therefore, the prediction power of the mathematical model is suspect. The assumption No. 2 seems highlyicredible. Such problem is severe given the non-robustness of the Nash (respectively the 
Bayes-Nash-Harsanyi) equilibrium, i.e. when sufficiently small changes in the game specification can change drastically the predicted outcomes of the game.

The subjective approach of the game proposed in this study attempts to present a more realistic mathematical model of strategic interactions (or conflicts), where each player takes only a subjective partial view of his/her individual decision. We consider a subjective strategic game that involves incomplete information and analyze the relationship between layers' subjective images of game and the realized outcomes. Under suitable assumptions, we prove the existence of subjective Nash equilibrium. Each player makes his/her best response based on a personal belief, that is, a subjective strategy profile. Each player's belief is justified only by realized outcomes. The player's rationality is represented basically by the best response behavior. Each player chooses a behavior by considering the consistency of his/her own subjective solution (of the game) concept with realized outcome. Each player simultaneously chooses both a personal strategy and a required belief regarding opponents' subjective strategies and utility functions, in order to satisfy a personal view of solution (of the game) concept. In other words, each player is assumed to observe a realized outcome via some solution concept and some related subjective image of the game. Since the subjective approach makes weaker assumptions than the objective approach, its prediction power is weaker. For many applications, however, weaker but more reliable predictions seem preferable to sharp but less reliable ones. The subjective approach of the game leads to a natural subjective Nash equilibrium concept and to meaningful results. However, the subjective approach is still complicated in requiring the players to solve a Pareto optimization problem.

\section{Definition 1.}

A subjective game is a game where other players' strategies and utility functions are conjectured by each player.

A subjective game has subjective strategies an utility functions based on subjective information of each player.

There is still the question of the choice of the appropriate players' solution concept of the subjective game. We will adopt the Nash equilibrium as the players' subjective solution concept.

\section{Definition 2.}

Nash equilibrium of an n-person game is a vector of behavior strategies

$$
f=\left(f_{1}, f_{2}, \ldots, f_{i}, \ldots, f_{n}\right)
$$

where each players' strategy $f_{i}$ being a best response to his/her opponents' strategies. That is, $f$ maximizes the utility function $u_{i}=u_{i}(f, e)$ of each player I, where e denotes the environment response function. 
A subjective rational player chooses his/her strategy $f_{i}$ to best response to his/her individual beliefs about his/her opponents' strategies. The players are assumed to be subjectively rational. Except for knowing his/her utility functions are not necessarily correct, nor do they coincide with other players' assessments.

A vector of subjectively rational strategies $f$ can be in subjective equilibrium if the play it induces coincides (with probability 1) with the plays induced by the beliefs of each player $\mathrm{i}=1,2 \ldots, \mathrm{n}$ as described by his/her beliefs vector $f_{\mathrm{i}}$. We think of a subjective equilibrium as being stable with respect to dynamic (and stochastic) process of learning and with respect to Pareto optimization. Players placed at such a subjective equilibrium will not alter their beliefs and will have no incentive to alter their subjective strategies.

Notions of "Subjective Equilibrium" are not new in economic sciences and game theory (see Battigalli et al., 1992, for a survey). Hahn (1973) assumed that players maximize their expected utility relative to their subjective hypothesis about the future evolution of the economy. Fundenberg and Lebine (1993) introduced the notion of "self-confirming equilibrium" for finite subjective games. Each player in such a game chooses a subjective strategy to maximize his/her expected utility function, given his/her subjective beliefs about opponents' strategies. These beliefs allow the possibility that the opponents' strategies are positively (or negatively) correlated.

The notion of subjective equilibrium considered in our study emerges in a dynamic learning process of $\mathrm{n}$ players engaged in a strategic game. Each player in the model chooses a strategy to maximize the mathematical expectation of utility function, given his/her subjective beliefs about the strategies of his/her opponents.

The main results of our study are the following:

\section{Theorem 1.}

Under an assumption of compatibility of the subjective beliefs of the players with the truth (or under an assumption of compatible beliefs in the sense of KolmogorovSmirnov statistics), after sufficiently long time (e.g., after 5 years), the players must play a subjective $\varepsilon$-Nash equilibrium, for arbitrary $\varepsilon>0$. At such equilibrium, the beliefs each player holds about the evolution of the game approximately coincide with the objective Nash equilibrium.

This fundamental result stated that there exists a dynamic process of learning that leads subjectively rational players to subjective Nash equilibrium. While subjective Nash equilibrium is reached as the limit of such random processes, only $\varepsilon$-subjective equilibrium is attained in finite times. For this reason, it is important to study the behavior of the players induced by these subjective equilibria, and to compare it to the better-known counterparts, objective Nash and/or $\varepsilon$-Nash equilibrium.

\section{Theorem 2.}

A behavior of subjectively rational players induced by a subjective $\varepsilon$ - equilibrium must be close to a behavior induced by an objective $\varepsilon$-equilibrium. 
An objective $\varepsilon$-Nash equilibrium requires for each player to choose a strategy that is $\varepsilon-$ best response against the precise strategies used by his/her opponents. That is, his/her utility function should be within $\varepsilon$-neighbor of the Pareto optimally possible strategy against his/her opponents. On the other hand, the subjective $\varepsilon-N a s h$ equilibrium requires precise Pareto optimization against beliefs of the players that are almost accurate (or accurate with probability $1-\varepsilon$ )

\section{Theorem 3.}

The mathematical expectation of subjective Pareto optimal strategies of experts converges in probability to a subjective $\varepsilon-$ Nash equilibrium, for arbitrary $\varepsilon>0$.

Some features of the mathematical results should be emphasized:

1. The players' objective is to maximize, relative to their individual subjective beliefs, mathematical expectation of their utility functions. Learning is not a goal in itself here but is, rather, consequence of overall individual utility function maximization plans. Learning is acquired as the real game process. In this sense it may be thought of as "learning by playing", paralleling well-known Kenneth Arrow's "learning by doing".

2. Learning takes place through Bayesian updating of the individual prior beliefs of players, following the approach of games of incomplete (or imperfect) information, see Harsanyi (1967).

3. We depart from the standard assumptions of Game Theory by not requiring that all players have full knowledge of each-others' utility functions or strategies. We replace these standard assumptions by a weaker assumption of compatibility of beliefs with the truth. This assumption requires that all players' subjective beliefs do not assign zero subjective probability (in the sense of von-Mizes and Kolmogorov) to random events that can occur in the play of the strategic game.

\section{Application in a scholarship strategy in a private university (i.e., UNYT)}

We will apply Theorems 1, 2 and 3 of the section 3 to develop a subjective Nash optimal scholarship strategy of a random private university (UNYT during the academic year 2009-2010.) We emphasize that the quality of enrolled students is a very important component of any private university's strategy. The players of the strategic game are shareholders and the academic staff, the enrolled students and other private universities inAlbania. (see Table 1 regarding the main actual compettors of UNYT).

The independent individuals' proposals of a subset of the academic staff for discount rate of enrolled students in UNYT during the year 2009-2010 are given in Table 2. The proposed discount rate depends on the High School GPA of the enrolled student. We consider two cases: $8 \leq G P A<9,9 \leq G P A \leq 10$.

The sample size is $\mathrm{n}=27$. We compute the sample mean $\bar{x}$ and sample standard deviation $\mathrm{s}$. 
First case: $\quad \bar{x}=22.9 \%$ and $\mathrm{s}=7.4 \%$

Second case: $\quad \bar{x}=39 \%$ and $\mathrm{s}=13.3 \%$

Several experts in Game Theory are assumed to conjecture that realized outcome of the subjective game should be Pareto efficient. That is, each player of the game considers not only his/her private outcome (profit) but also social outcome (profit). The experts' sample size is $n=7$. We calculate the sample mean $\overline{\mathrm{x}}$ and sample standard deviation $\mathrm{s}$.

$\begin{array}{lccc}\text { First case: } & (8 \leq G P A<9) & \bar{x}=22.1 \% & \mathrm{~s}=2.7 \% \\ \text { Second case: } & (9 \leq G P A \leq 10) & \bar{x}=38.6 \% & \mathrm{~s}=6.3 \%\end{array}$

Therefore, we propose the following discount rates for enrolled students in this particular private university (UNYT) during the academic year 2009-2010:

First case: $\quad(8 \leq G P A<9) \quad 22 \%$ or $23 \%$

Second case: $\quad(9 \leq G P A \leq 10) \quad 39 \%$

This proposal represents an advice to all the players in the game. (i.e., to advice each player what strategy to choose). Any advice that is not a Nash equilibrium would have the unsettling property that there would always be some players for whom the advice was bad. If all other players followed the advice directed to them, it would be better for some players to do differently from what they were advised. If the advice is a Nash equilibrium, however, this will not be the case, because the advice to each player is the best response to the advice given to the other players. This point of view is also used to derive predictions of the players' behavior, if they can be approximated as rational players. A Nash equilibrium is a self-enforcing (implicit or explicit) agreement that, once reached by the layers, does not need any external means of enforcement, because it is in the selfinterest of each player to follow the agreement, if the others do so.

\section{Conclusion}

In this study we have applied the Nash equilibrium approach and Bayes- NashHarsanyi equilibrium approach to investigate the process of enrolled students in private universities, assuming that the high school GPA of the students is between 8 and 9 or between 9 and 10 .

Based on the individual independent proposals on $n=27$ members of the actual staff of UNYT (from these only 7 are experts in the Game Theory) we suggested the following discount rate for enrolled students during academic year 2009 2010:

If $8 \leq \mathrm{GPA}<9$, then discount rate is $23 \%$ 
If $9 \leq \mathrm{GPA} \leq 10$, then discount rate is $39 \%$

This suggestion is based on the subjective definition of probability and on subjective Nash equilibrium of a finite strategic game with random demand, where "demand" represents "the number of enrolled students in UNYT during an arbitrary academic year".

According to R. Von Mizes, A. Ya. Hinchin and A. N. Kolmogorov, if there is little or no past experience on which to base the probability of a random event, a probability may be arrived at subjectively. Essentially, this means evaluating the individual opinions of experts and other subjective information, and then arriving at the probability.

Following Kolmogorov's Central Limit Theorem (or Kolmogorov - Chaptain's Theorem), we can assume that the demand is approximately by a normal (or lognormal) random variable.

In a competitive universitary market with random demand, monitoring is more difficult and complicated.

We hope that the proposal for discount rate of enrolled students in private universities will have a significant positive impact in several fields (economic, social, academic, competitive and psychological).

Our mathematical models consider two key components of the actual universitary educational process in the Republic of Albania.

- First, students differ in their ability. We assume that higher ability increases the enrolled student's educational achievement.

- Second, householders differ in their incomes, with higher income increasing the demand for universitary educational achievement.

A part of the students in our mathematical models is characterized by a conflict between the individual ability and household income.

The optimal solution for this conflict is the Nash equilibrium or Bayes- NashHarsanyi equilibrium, over the strict hierarchy of private universities' quality in Tirana. The main results of our study are Theorem 1, Theorem 2, and Theorem 3. However, the uniqueness of equilibrium does not hold.

\section{References}

K. J. Arrow, The economic implications of learning by doing, Review of Economic Studies, 29, 1962, 155-173.

R. J. Aumann, Subjectivity and correlation in randomized strategies, J. Math. Econ. 1, 1974, 67-96. 
D. Fundenberg and J. Tirole, Game Theory, MIT Press, 1991.

D. Fundenberg and D. K. Levine, Self-Confirming equilibrium; Econometrica 61, 1993, 523-545.

D. Fundenberg and D. K. Levine, Theory of Learning in Games, Harvard University 1996.

F. Hahn, On the notion of equilibrium in economics: Inaugural Lecture, Cambridge: Cambridge University Press, 1973.

J. C. Harsanyi, Games with incomplete information played by Bayesian players, Management Science 14, 99 159-182, 320-334, 486-502, 1973.

F. A. Von Hayek, The Pretence of Knowledge, Nobel Memorial Lecture, 1974.

O. Hidetugu, Rationalized subjective equilibria in repeated games, the Canadian Journal of Economics No 1, 2003, 168-1941.

C. A. Holt, A. E. Roth and V. L. Smith, The Nash Equilibrium: a perspective, Proc. Nat. Acad. Sci. USA, Vol 101, 2004, 3999-4002.

E. Kalai and E. Lehrer, Subjective Equilibrium in repeated games, Econometrica 61, 1993, 1231-1240.

E. Kalai and E. Lehler, Subjectice games and equilibria, Games and Economic Behavior, $8,1995,123-163$.

E. Kalai, Large robust games, Econometrica, Vol 72, No 2, 2004, 1631-1665.

N. Kolmogorov, Selected Works (in Russian), Nauka, Moscow, Vol I (1985), Vol II (1986), Vol III (1987) and Vol IV (1988).

R. B. Myerson, Nash equilibrium and the history of economic theory, Journal of Economic Literature 37(3), 1999, 1067-1082.

J. Nash, The Bargaining problem, Econometrica 18(2), 1950, 155-162.

J. Nash, Equilibrium points in n-person games, Prod. Nat. Acad. Sci. USA, Vol 36, 1951, 48-49

J. Nash, Non-cooperative games, Annals Mat. Vol. 54, 1953, pp 289-295.

G. Owen, Game Theory, Academic Press, 1995.

A. Roth, Axiomatic models of bargaining, Springer0Verlag, 1979.

R. Selten, Reexamination of the perfectness concept for equilibrium point, Int. J. Game Theory, Vol. 4, 1975, pp 25-55.

D. Zhang, A logical model of Nash bargaining solution, working paper, 2007. 
